\title{
Two-portal Dark Matter
}

\author{
Karim Ghorbani * \\ Physics Department, Faculty of Sciences, Arak University, Arak 38156-8-8349, Iran \\ Hossein Ghorbani ${ }^{\dagger}$ \\ Institute for Research in Fundamental Sciences (IPM) \\ School of Particles and Accelerators, P.O. Box 19395-5531, Tehran, Iran
}

\begin{abstract}
We propose a renormalizable dark matter model in which a fermionic dark matter (DM) candidate communicates with the standard model particles through two distinct portals: Higgs and vector portals. The dark sector is charged under a $U(1)^{\prime}$ gauge symmetry while the standard model has a leptophobic interaction with the dark vector boson. The leading contribution of DM-nucleon elastic scattering cross section begins at one-loop level. The model meets all the constraints imposed by direct detection experiments provided by LUX and XENON100, observed relic abundance according to WMAP and Planck, and the invisible Higgs decay width measured at the LHC. It turns out that the dark matter mass in the viable parameter space can take values from a few $\mathrm{GeV}$ up to $1 \mathrm{TeV}$. This is a new feature which is absent in the models with only one portal. In addition, we can find in the constrained regions of the parameter space a DM mass of $\sim 34 \mathrm{GeV}$ annihilating into $b$ quark pair, which explains the Fermi-LAT gamma-ray excess.
\end{abstract}

*kghorbani@ipm.ir

${ }^{\dagger}$ pghorbani@ipm.ir 


\section{Introduction}

Cosmological observations indicate unequivocally that the Standard Model (SM) particles constitute only $5 \%$ of the mass content of our Universe, the rest $26 \%$ dark matter (DM) and 69\% dark energy are yet unknown [1,2]. If the weakly interacting massive particle (WIMP) is a correct scenario for DM, a big question however, is what the dark matter is made of and what would be the fundamental interaction of its constituents with the ordinary matter. Direct detection experiments are designed to probe dark matter (DM) elastic scattering off nuclei. In this regards, underground LUX [3] and XENON100 [4] dark matter experiments so far have found no signal on these type of interactions, even though they provide us with an upper limit on the elastic scattering cross section.

Recent observation of the galactic center gamma-ray excess (GCE), given its intensity and spatial morphology, can be explained by the dark matter annihilation in the galactic center (GC) [5-11]. There is a large number of models in the literature suggested in order to explain the gamma-ray excess but among them are models with dark matter, annihilating predominantly into $b$ quark pair and on top of that can evade stringent bounds from direct detection experiments [12 18].

Moreover, there are models as extensions to the SM with an additional $U(1)^{\prime}$ gauge symmetry and its associated $Z^{\prime}$ boson which contain a DM candidate annihilating via an intermediate neutral gauge boson $Z^{\prime}$ [19 29].

Models with an extra broken $U(1)^{\prime}$ gauge symmetry are motivated by new physics beyond the SM, as examples for various models we recall, those focusing on the grand unified theories like $S O(10)$ and $E_{6}$, (see e.g. 30, 31] and for a review consult [32]), dynamical symmetry breaking models like topcolor (see [33] for a review), decoupled models like leptophobic $Z^{\prime}$ [34], Little Higgs theories [35 38], Twin Higgs model [39], family non-universal scenario [40].

There are many models where the SM and DM sectors interact through only one portal, in the sense that there is only one type of mediator (e.g. scalar or vector) to connect the two sectors. There are also models with two similar mediators [41,42].

In this work we propose for the first time a minimal dark matter model with two distinct portals in tandem: Higgs and vector ones. In this article we construct a two-portal DM model based on an extra gauge symmetry $U(1)^{\prime}$ which not only can explain the observed relic density and the galactic gamma-ray excess but also can evade direct detection as well as the constraints from invisible Higgs decay.

In our two-portal model the dark sector consists of a Dirac fermion as a WIMP dark matter candidate, a complex scalar field as the first mediator, both of them charged under a new $U(1)^{\prime}$ gauge symmetry. Obviously the fermion dark matter is coupled to the dark gauge boson $Z^{\prime}$, covariantly. In addition, we assume that only the SM quarks are also charged under the $U(1)^{\prime}$. Thus, the $Z^{\prime}$ field interacts with the SM particles and the DM, hence the second mediator of the model. In the present model we will deal with a non-universal $Z^{\prime}$ gauge boson, such that the new gauge boson has negligible coupling to the first and second generation of the SM quarks.

Models with a new $Z^{\prime}$ gauge boson which prefer interaction with only the third family of the SM fermions are widely investigated within different scenarios beyond 
the SM. Among these scenarios, we recall superstring inspired models [34, topcolor assisted technicolor model [43, phenomenology of a $Z^{\prime}$ boson coupled only to thirdfamily fermions [44, electroweak constraints on models with non-universal $Z^{\prime}$ bosons [45], and warped models in which the extra $Z^{\prime}$ boson typically couples only to the third generation [23].

The present article has the following structure. In the next section we introduce our model in detail. In Sec. 3 we compute the SM Higgs invisible decay width within the model and address the constraints on the invisible decay width from the LHC measurements. We derive a formula for the DM-nucleon cross section in Sec. 4. In Sec. 5 we discuss on the DM relic density within the thermal freeze-out mechanism and our numerical computations for the relic abundance and DM-nucleon interaction are discussed in Sec. 6. In Sec. 7 we will find regions in the viable parameter space of the two-portal model that can explain the Galactic gamma-ray excess given the recent Fermi-LAT data analysis. We finally finish with a conclusion.

\section{The Model}

We introduce a dark matter model which has the property of having DM-SM interaction through two different portals, i.e. the vector portal and the Higgs portal. We will show in Sec. 4 that for the model to be elaborated below there is no tree level DM scattering off nuclei and the Feynman diagrams begin with a one-loop contribution which turns out to be suppressive. A two-portal dark matter model can therefore be designed in order to predict the DM elastic scattering to be consistent with the direct detection experiments. This is reminiscent to the velocity suppressed models for elastic scattering processes but in a different way.

The details of the model comes in the following: beside having a scalar field that mixes with the SM Higgs via the Higgs portal, to have a vector portal interaction, we assume a $U(1)^{\prime}$ gauge theory in the dark sector as the simplest model including a gauge boson. We also assume that only the SM quarks (but not the leptons) are charged under the $U(1)^{\prime}$. In other words, we are dealing with a leptophobic vector portal interaction.

The total Lagrangian consists of the standard model part, the dark sector and the interactions between these two sectors:

$$
\mathcal{L}=\mathcal{L}_{\mathrm{SM}}+\mathcal{L}_{\mathrm{DM}}+\mathcal{L}_{\text {int }}
$$

The SM covariant derivative acting on the quarks must now be modified as

$$
D_{\mu}^{\mathrm{SM}} \rightarrow D_{\mu}^{\mathrm{SM}}=D_{\mu}^{\mathrm{SM}}-i g^{\prime} \frac{z}{2} Z_{\mu}^{\prime},
$$

where $z$ is the dark charge of the quark field that the covariant derivative acts on.

The dark matter Lagrangian consists of a fermionic dark matter and a complex scalar field both charged under $U(1)^{\prime}$ :

$$
\mathcal{L}_{\mathrm{DM}}=-\frac{1}{4} F_{\mu \nu}^{\prime} F^{\prime \mu \nu}+\bar{\chi}\left(i \gamma^{\mu} D_{\mu}^{\prime}-m_{\chi}\right) \chi+\left(D_{\mu}^{\prime} \phi\right)\left(D^{\prime \mu} \phi\right)^{*}-m_{\phi}^{2}\left(\phi \phi^{*}\right)-\frac{1}{4} \lambda\left(\phi \phi^{*}\right)^{2},
$$


where $F^{\prime \mu \nu}$ is the $U(1)^{\prime}$ field strength, $\chi$ is a Dirac fermion as the dark matter candidate and $\phi$ is a complex scalar field. The dark sector covariant derivative is given by

$$
D_{\mu}^{\prime}=\partial_{\mu}-i g^{\prime} \frac{z}{2} Z_{\mu}^{\prime}
$$

We assume that the interaction of the standard model particles with the $U(1)^{\prime}$ gauge boson, $Z^{\prime}$ is leptophobic. In other words, non of the leptons in the SM are charged under $U(1)^{\prime}$. The $\mathcal{L}_{\text {int }}$ consisting of the scalar-Higgs and $Z^{\prime}$-quark interactions reads:

$\mathcal{L}_{\text {int }}=-\lambda\left(\phi \phi^{*}\right)\left(H H^{\dagger}\right)+g^{\prime} \frac{z_{Q_{L}}}{2} Z_{\mu}^{\prime} \bar{Q}_{L} \gamma^{\mu} Q_{L}+g^{\prime} \frac{z_{u_{R}}}{2} Z_{\mu}^{\prime} \bar{u}_{R} \gamma^{\mu} u_{R}+g^{\prime} \frac{z_{d_{R}}}{2} Z_{\mu}^{\prime} \bar{d}_{R} \gamma^{\mu} d_{R}$,

where $H$ is the SM Higgs doublet, $Q_{L}, u_{R}$ and $d_{R}$ are respectively left-handed quark doublet, right-handed up-quark singlet and right-handed down-quark singlet. The couplings of the light quarks $u, d, \ldots$ with $Z^{\prime}$ are considered to be negligible. Therefore, by $z_{Q_{L}}, z_{u_{R}}$ and $z_{d_{R}}$ we mean the dark charge of only the third quark family i.e. the $t$ and $b$ quarks. The upshot is that the dark matter scattering off nuclei lacks the tree level contribution (see Fig. 4) and remains suppressed as expected from direct detection experiments.

Having introduced dark gauge boson, $Z^{\prime}$, interacting with SM and DM fermionic currents, one should note that new anomalies from triangle Feynman diagrams may arise. However, it can be shown that assigning appropriate dark charges for quarks can lead to a anomaly-free theory 1 .

The anomaly-free conditions put constraints on the top and bottom quark $U(1)^{\prime}$ charges: $z_{Q_{L}}=-2, z_{u_{R}}=+2$ and $z_{d_{R}}=+2$. Substituting these charges in Eq. (5) the $\mathcal{L}_{\text {int }}$ becomes:

$$
\mathcal{L}_{\mathrm{int}}=-\lambda_{1}\left(\phi \phi^{*}\right)\left(H H^{\dagger}\right)+g^{\prime} Z_{\mu}^{\prime} \bar{t} \gamma^{\mu} \gamma^{5} t+g^{\prime} Z_{\mu}^{\prime} \bar{b} \gamma^{\mu} \gamma^{5} b
$$

The $\mathcal{L}_{\text {int }}$ consists of the Higgs portal where the scalar field interacts with the SM Higgs quadratically, and the vector portal where the dark gauge boson interacts axially with the third family quarks.

The scalar field $\phi$ does not interact directly with the DM particle $\chi$ but interacts with that only through another mediator of the model i.e. the dark gauge boson $Z^{\prime}$. On the other hand, the gauge boson mediator interacts directly with the dark matter particle which can be seen by following the red line in Fig. 1. The scalar field $\phi$ and the gauge boson $Z^{\prime}$ are connected to the SM respectively via the Higgs portal and via an interaction with the third family of quarks (vector portal). The novelty of the current minimal model is that there are two distinct mediators at the same time which makes a bridge between the DM sector and the SM sector. Schematically these interactions are shown in Fig. 1.

\footnotetext{
${ }^{1}$ For some details on anomaly-free conditions in the extended SM including $U(1)^{\prime}$ interactions with no additional fermions see 46. In our model we have an additional fermion that is the dark matter Dirac field. Taking equal dark charges for left- and right-handed components of the Dirac fermion $z_{\chi_{L}}=z_{\chi_{R}}$ we lead to the same anomaly-free conditions mentioned in [46].
} 


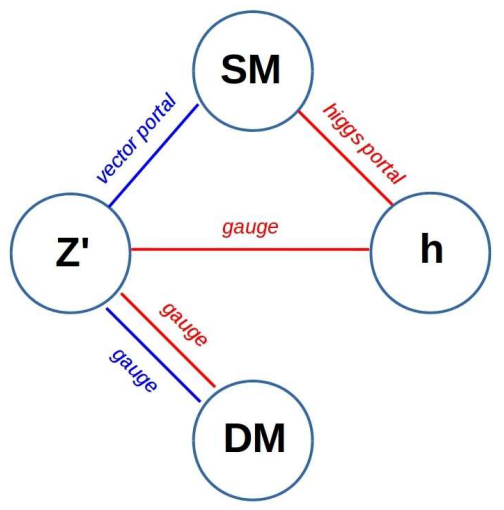

Figure 1: Two-portal SM-DM interactions: the dark matter candidate indirectly can interact with standard model through a Higgs and a vector portal. In this model the dark matter can interact directly with only one of the mediators i.e. the dark vector boson.

The SM Higgs potential is given by

$$
V_{H}=-\mu_{H}\left(H H^{\dagger}\right)-\lambda_{H}\left(H H^{\dagger}\right)^{2}
$$

where the Higgs doublet takes on a non-zero vacuum expectation value (vev),

$$
H=\frac{1}{\sqrt{2}}\left(\begin{array}{c}
0 \\
v+\tilde{h}
\end{array}\right)
$$

We assume that the scalar mediator also takes a non-zero vev,

$$
\langle\phi\rangle=v^{\prime} \Rightarrow \phi=v^{\prime}+\frac{1}{\sqrt{2}} \tilde{h}^{\prime} .
$$

$\tilde{h}$ and $\tilde{h}^{\prime}$ are respectively the SM Higgs and the singlet scalar field fluctuations around their vacuum expectation values. It is worth mentioning that once the complex scalar develops a non-zero vacuum expectation value, $v^{\prime}$, this breaks the $U(1)^{\prime}$ symmetry spontaneously and the $Z^{\prime}$ boson will acquire mass. On top of that, the strength of these vertices, $Z^{\prime} Z^{\prime} h$ and $Z^{\prime} Z^{\prime} h^{\prime}$, is proportional to $v^{\prime}$. Therefore, according to the Feynman diagram in Fig. 2, the choice $v^{\prime}=0$ will give rise to zero DM-quark elastic scattering cross section which is a trivial scenario.

After substituting Eq. (91) in Eq. (3) and expanding the Lagrangian, the mass of the dark gauge boson turns out to be $g^{\prime} v^{\prime} / \sqrt{2}$. As may be followed in [16] the masses of the SM Higgs particle $h$ and the scalar mediator $h^{\prime}$ can be obtained by diagonalizing the mass matrix,

$$
M=\stackrel{\tilde{h}}{\tilde{h}} \begin{array}{cc}
\tilde{h}^{\prime} \\
\tilde{h}^{\prime}
\end{array}\left(\begin{array}{cc}
2 \lambda_{H} v^{2} & \sqrt{2} \lambda_{1} v v^{\prime} \\
\sqrt{2} \lambda_{1} v v^{\prime} & \frac{1}{2} \lambda v^{\prime 2}-\frac{1}{2} \lambda_{1} v^{2}
\end{array}\right),
$$


where we have used the following relations coming from minimizing the total potential,

$$
\begin{gathered}
m_{\phi}^{2}=-\lambda v^{\prime 2}-\lambda_{1} v^{2}, \\
\mu_{H}^{2}=-\lambda_{H} v^{2}-\lambda_{1} v^{\prime 2} .
\end{gathered}
$$

We can redefine the scalars $\tilde{h}$ and $\tilde{h}^{\prime}$ by introducing a mass mixing angle in order to get a diagonalized mass matrix,

$$
\begin{aligned}
& h=\sin (\theta) \tilde{h}+\cos (\theta) \tilde{h}^{\prime}, \\
& h^{\prime}=\cos (\theta) \tilde{h}-\sin (\theta) \tilde{h}^{\prime},
\end{aligned}
$$

with the mixing angle $\theta$ being

$$
\tan (\theta)=\frac{1}{1+\sqrt{1+y^{2}}}, \quad y=\frac{2 m_{\tilde{h} \tilde{h}^{\prime}}^{2}}{m_{\tilde{h}}^{2}-m_{\tilde{h}^{\prime}}^{2}} .
$$

where $m_{\tilde{h} \tilde{h}^{\prime}}$ is the off-diagonal entry of the mass matrix in Eq. (10).

The masses of the redefined scalar fields read,

$$
m_{h}^{2}, m_{h^{\prime}}^{2}=\frac{m_{\tilde{h}}^{2}+m_{\tilde{h}^{\prime}}^{2}}{2} \pm \frac{m_{\tilde{h}}^{2}-m_{\tilde{h}^{\prime}}^{2}}{2} \sqrt{1+y^{2}}
$$

where the upper sign (lower sign) corresponds to $m_{h}\left(m_{h^{\prime}}\right)$. The standard model Higgs is denoted here by $h$ with mass $m_{h}=125 \mathrm{GeV}$ and $h^{\prime}$ is the singlet scalar. Exploiting Eq. (15) and Eq. (16) we can obtain the quartic couplings as a function of SM Higgs mass, singlet scalar mass, the mixing angle and the vacuum expectation values $v$ and $v^{\prime}$,

$$
\begin{array}{r}
\lambda_{H}=\frac{m_{h^{\prime}}^{2} \sin ^{2} \theta+m_{h}^{2} \cos ^{2} \theta}{2 v^{2}}, \\
\lambda=\frac{m_{h^{\prime}}^{2} \cos ^{2} \theta+m_{h}^{2} \sin ^{2} \theta}{v^{2} / 2}-\frac{v^{2}}{v^{\prime 2}} \lambda_{1}, \\
\lambda_{1}=\frac{m_{h}^{2}-m_{h^{\prime}}^{2}}{2 \sqrt{2} v v^{\prime}} \sin 2 \theta .
\end{array}
$$

The vacuum stability of the total potential is equivalent to having positive eigenvalues for the scalar boson mass-squared matrix. At tree-level this brings in the following constraints on the couplings (see also [16]); $\lambda_{H}>0, \lambda v^{\prime 2}>\lambda_{1} v^{2}$ and $v^{\prime 2}\left(\lambda_{H} \lambda-2 \lambda_{1}^{2}\right)>$ $v^{2} \lambda_{1} \lambda_{H}$. In our numerical analysis we will choose $m_{\chi}, m_{h^{\prime}}, \theta, v^{\prime}$ and $g^{\prime}$ as free parameters. 


\section{$3 \quad$ Invisible Higgs Decay}

In the present model, there are two new decay channels for the SM Higgs which can modify the total decay width of the Higgs boson within the SM. The current measurement of total decay width for the $125 \mathrm{GeV}$ Higgs reads, $\Gamma_{\mathrm{Higgs}}^{\mathrm{SM}} \sim 4 \mathrm{MeV}$ [47]. In case the dark gauge boson is light enough such that $m_{Z^{\prime}}<m_{\mathrm{h}} / 2$ the Higgs boson is kinematically allowed to undergo the following invisible decay

$$
\Gamma_{\mathrm{inv}}\left(h \rightarrow Z^{\prime} Z^{\prime}\right)=\frac{v^{\prime 2} g^{\prime 4} \sin ^{2} \theta}{16 \pi m_{h}}\left(1-4 m_{Z^{\prime}}^{2} / m_{h}^{2}\right)^{1 / 2} .
$$

In addition when we consider light scalar boson with $m_{h^{\prime}}<m_{h} / 2$, another decay channel is plausible for the SM Higgs with

$$
\Gamma_{\text {inv }}\left(h \rightarrow h^{\prime} h^{\prime}\right)=\frac{c^{2}}{128 \pi m_{h}}\left(1-4 m_{h^{\prime}}^{2} / m_{h}^{2}\right)^{1 / 2},
$$

where

$$
\begin{array}{r}
c=3 \sqrt{2} \lambda v^{\prime} \cos ^{2} \theta \sin \theta+12 \lambda_{H} v \cos \theta \sin ^{2} \theta-6 \lambda_{1} v \cos \theta \sin ^{2} \theta \\
+2 \lambda_{1} v \cos \theta+6 \sqrt{2} \lambda_{1} v^{\prime} \sin ^{3} \theta-4 \sqrt{2} \lambda_{1} v^{\prime} \sin \theta .
\end{array}
$$

We thus expect the total Higgs decay width to modify as

$$
\Gamma_{\text {Higgs }}^{\text {tot }}=\cos ^{2} \theta \Gamma_{\text {Higgs }}^{\mathrm{SM}}+\Theta\left(m_{h}-2 m_{Z^{\prime}}\right) \Gamma\left(h \rightarrow Z^{\prime} Z^{\prime}\right)+\Theta\left(m_{h}-2 m_{h^{\prime}}\right) \Gamma\left(h \rightarrow h^{\prime} h^{\prime}\right),
$$

where $\Theta$ is the step function. It is worth mentioning that since here Higgs has no tree level interaction with the DM, the invisible Higgs decay width is independent of DM mass at leading order. There exist an experimental upper limit for the invisible branching ratio of the $125 \mathrm{GeV}$ Higgs decay investigated at the $\mathrm{LHC}, \mathrm{Br}_{\text {inv }} \lesssim 0.35$ [48]. In our numerical analysis when applicable, we restrict ourself into the parameter space which satisfies the condition $\Gamma_{\text {inv }} / \Gamma_{\text {Higgs }}^{\text {tot }} \lesssim 0.35$.

\section{Direct Detection}

The tree level DM-quark elastic scattering is suppressed because dark vector boson interaction with light quarks are assumed to be negligible. As depicted in Fig. 2 the first leading contribution to the elastic scattering amplitude is obtained through a one-loop interaction coupled to the SM Higgs or the Higgs-like scalar where the DM particle and dark gauge boson run in the loop. The DM-quark scattering amplitude is obtained as

$$
\begin{array}{r}
\mathcal{M}=-4 i g^{\prime 4} v^{\prime} \frac{m_{q}}{v}\left[\frac{\sin ^{2} \theta}{\left(p_{1}-p_{2}\right)^{2}-m_{h}^{2}}-\frac{\cos ^{2} \theta}{\left(p_{1}-p_{2}\right)^{2}-m_{h^{\prime}}^{2}}\right] \bar{q} q \times \\
\int \frac{d^{4} q}{(2 \pi)^{2}} \frac{\bar{\chi}\left(p_{2}\right) \gamma_{\mu}\left(\not 1+m_{\chi}\right) \gamma^{\mu} \chi\left(p_{1}\right)}{\left[\left(p_{2}-q\right)^{2}-m_{Z^{\prime}}^{2}\right]\left[\left(p_{1}-q\right)^{2}-m_{Z^{\prime}}^{2}\right]\left[q^{2}-m_{\chi}^{2}\right]},
\end{array}
$$




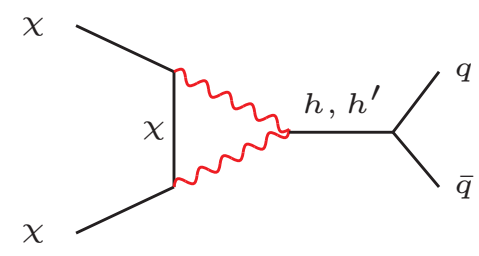

Figure 2: The Feynman diagram for the DM elastic scattering with quarks. The wavy lines stand for the propagation of the $Z^{\prime}$ boson.

where respectively $p_{1}$ and $p_{2}$ are the four-momenta of the incoming and outgoing DM, and the DM four-momentum in the loop is denoted by $q$. Since $\left(p_{1}-p_{2}\right)^{2} \ll m_{\chi}^{2}, m_{Z^{\prime}}^{2}$, we can then perform the loop integral at $t=\left(p_{1}-p_{2}\right)^{2} \sim 0$ to get the effective scattering amplitude 2

$$
\mathcal{M}_{\mathrm{eff}}=\frac{g^{\prime 4} v^{\prime}}{4 \pi^{2} m_{\chi}} S(\beta) \frac{m_{q}}{v}\left[\frac{\cos ^{2} \theta}{m_{h^{\prime}}^{2}}-\frac{\sin ^{2} \theta}{m_{h}^{2}}\right](\bar{q} q)(\bar{\chi} \chi) \equiv \alpha_{q}(\bar{q} q)(\bar{\chi} \chi),
$$

where

$$
S(\beta)=-2+\beta \log \beta-\frac{\beta^{2}-2 \beta-2}{\sqrt{\beta^{2}-4 \beta}} \log \frac{\sqrt{\beta}+\sqrt{\beta-4}}{\sqrt{\beta}-\sqrt{\beta-4}},
$$

and $\beta=\left(\frac{m_{Z^{\prime}}}{m_{\chi}}\right)^{2}$. In order to find the DM-nucleon elastic scattering cross section one needs to evaluate the nucleonic matrix element. However, at the vanishing momentum transfer we can make use of the conventional assumption that the nucleonic matrix element with quark current is proportional to the nucleonic matrix element with nucleon current [49 51 ]

$$
\sum_{\mathrm{q}} \alpha_{q}\left\langle N_{f}|\bar{q} q| N_{i}\right\rangle \equiv \alpha_{N}\left\langle N_{f}|\bar{N} N| N_{i}\right\rangle
$$

in which

$$
\alpha_{N}=m_{N}\left(\sum_{q=u, d, s} f_{T q}^{N} \frac{\alpha_{q}}{m_{q}}+\frac{2}{27} f_{T g}^{N} \sum_{q=c, b, t} \frac{\alpha_{q}}{m_{q}}\right) .
$$

The scalar couplings $f_{T q}^{N}$ and $f_{T g}^{N}$ are responsible for the low energy strong interaction and nucleon mass is denoted by $m_{N}$. In the numerical computation in Sec. [6] we shall use the following values for the scalar couplings [52]

$$
f_{u}^{p}=0.0153, \quad f_{d}^{p}=0.0191, \quad f_{s}^{p}=0.0447 .
$$

Spin-independent (SI) total cross section of DM-nucleon elastic scattering is finally achieved as

$$
\sigma_{\mathrm{SI}}^{\mathrm{N}}=\frac{4 \alpha_{N}^{2} \mu_{\chi N}^{2}}{\pi},
$$

where $\mu_{\chi N}$ is the reduced mass of the DM-nucleon system.

\footnotetext{
${ }^{2}$ Currently, the typical recoil energy under examination at direct detection experiments is $E_{R} \sim 10$ $\mathrm{KeV}$. On the other hand, the momentum transferred to a nucleus of mass $M_{N}$ is given by $t=2 M_{N} E_{R}$. For a xenon nucleus for example, we obtain $t \sim 2 \times 10^{-3} \mathrm{GeV}^{2}$. Thus we expect $t \ll m_{\chi}^{2}, m_{Z^{\prime}}^{2}$.
} 

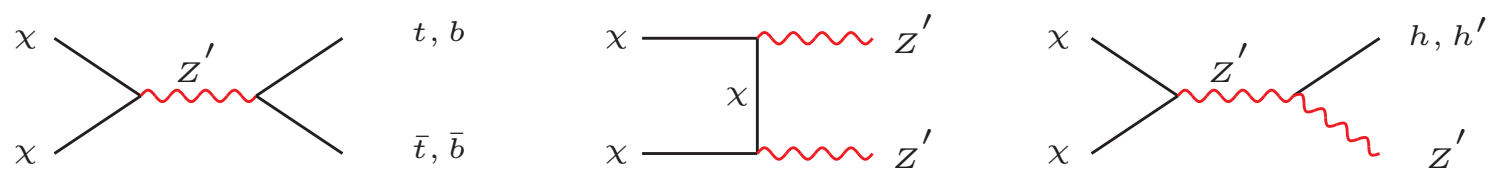

Figure 3: The Feynman diagrams for the DM annihilation processes.

\section{Relic Abundance}

The fermionic dark matter candidate in the model laid out earlier is of WIMP type DM whose present day density, the so called relic density, is a remnant from freeze-out epoch in the early Universe. The freeze-out mechanism is based on the assumption that dark particles had been in thermal equilibrium in the early time at temperatures $T \gtrsim m_{\mathrm{DM}}$. In an expanding Universe the annihilation rate of dark particles into SM particles slows down and there is an epoch with $T \ll m_{\mathrm{DM}}$ after which this rate descends below the Hubble expansion rate. On the other hand, from this time on dark particles are not kinematically allowed to get reproduced. Thus, in effect, the number density of dark particles, $n_{\chi}$, remains asymptotically constant within the comoving volume.

The leading DM annihilation reactions which are necessary to determine the relic density are shown in Fig. 3. In this figure the first and third annihilation processes occur via a $Z^{\prime}$ boson exchange in s-channel: $\chi \chi \rightarrow \bar{b} b, \bar{t} t, Z^{\prime} h, Z^{\prime} h^{\prime}$, while the second diagram shows annihilation with an intermediate $\mathrm{DM}$ via t- and $\mathrm{u}$-channel: $\chi \chi \rightarrow Z^{\prime} Z^{\prime}$. The Boltzmann equation provides us with the evolution of DM number density in terms of thermal averaged annihilation cross sections $\left\langle\sigma_{\text {ann }} v_{\text {rel }}\right\rangle$ as

$$
\frac{d n_{\chi}}{d t}+3 H n_{\chi}+\left\langle\sigma_{\text {ann }} v_{\text {rel }}\right\rangle\left[n_{\chi}^{2}-\left(n_{\chi}^{\mathrm{EQ}}\right)^{2}\right]=0,
$$

where, $n_{\chi}^{\mathrm{EQ}}$ is the DM number density at equilibrium condition and $H$ is the Hubble parameter. In order to determine the present value of the number density and therefore the relic density one should solve numerically the Boltzmann equation at freeze-out condition which is when the dark particles are away from equilibrium.

We first implement our model into the program LanHEP [53] to give us all the basic vertices and Feynman rules of our model. Later on to analyze the DM relic density we employ the package MicrOMEGAs [52] which requires our output files from the LanHEP program. To check the validity of our model implementation into LanHEP, we utilize the program CalcHEP [54] using our LanHEP outputs to calculate the annihilation cross sections. From this we found agreements with our analytical calculations given in appendix $\mathrm{A}$ for the relevant annihilation cross sections.

\section{Numerical Analysis}

In this section we will find the viable region in the parameter space which respect observed relic density, invisible Higgs decay width measurement and constraints from 

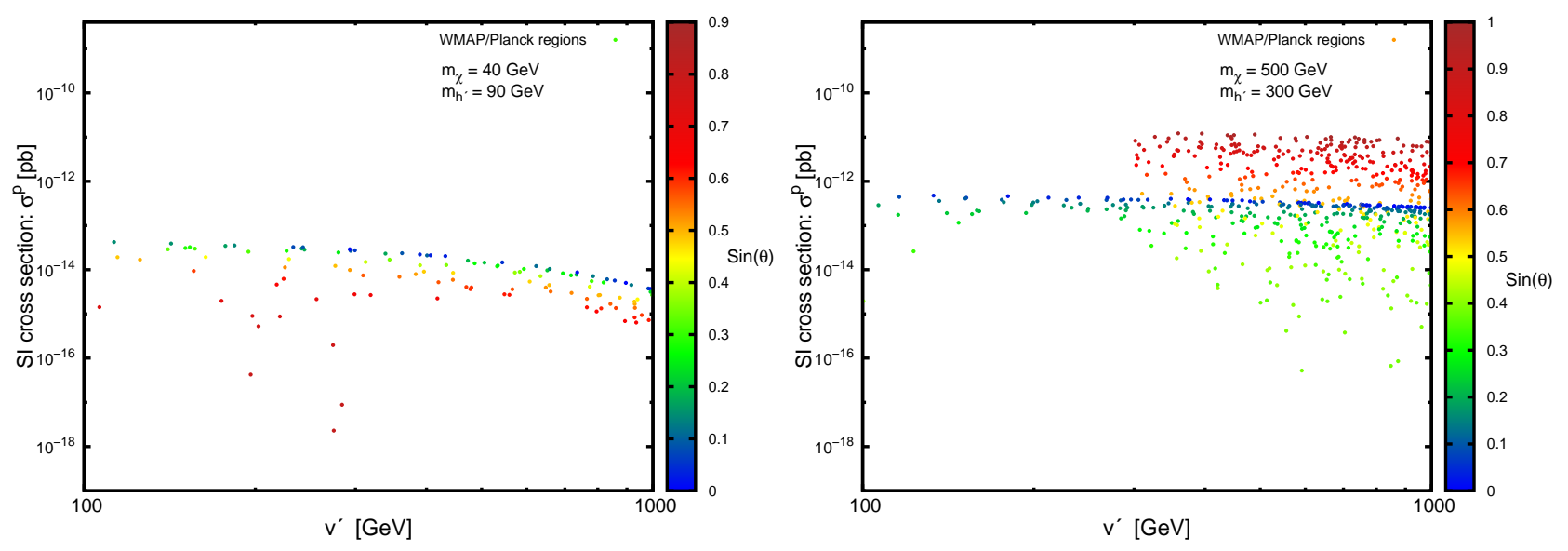

Figure 4: Results for the spin-independent elastic scattering cross section of DM with proton as a function of two variables, scalar vacuum expectation value $v^{\prime}$ and the mixing angle $\theta$. The coupling $g^{\prime}$ is generated in the range $0.001<g^{\prime}<1$. For both values of the DM mass, the elastic scattering cross section is below the upper limit given by LUX and XENON100.

direct detection experiments. We consider in our parameter space as independent free variables: $m_{\chi}, m_{h^{\prime}}, g^{\prime}, v^{\prime}$ and $\theta$. Throughout our study we keep fixed the SM Higgs mass as $m_{h}=125 \mathrm{GeV}$ and the SM Higgs vacuum expectation value as $v=246 \mathrm{GeV}$. As a first numerical look, we would like to find the viable region in the parameter space for a given set of variables $\left\{m_{\chi}, m_{h^{\prime}}\right\}$ by scanning over the scalar vacuum expectation value $v^{\prime}$ and the mixing angle $\theta$. To do so, we pick out random values for the coupling $g^{\prime}$ in the reasonable range of $0.001<g^{\prime}<1$. Our results presented in Fig. 4 for two sample values of the DM mass, indicate that it is possible to find viable regions in the parameter space for the wide range of $100<v^{\prime}<1000$ and the mixing angle $0<\theta<\pi / 2$.

To move on, we fix two variables out of five independent free parameters when we scan over the parameter space. For the vacuum expectation value of the singlet scalar we choose $v^{\prime}=800 \mathrm{GeV}$ and perform our calculations for two different mixing angle with $\sin \theta=0.01$ and $\sin \theta=0.1$. It is then ensured that with these choices and the range of the masses we will pick out for the singlet scalar, the quartic couplings will respect bounds from perturbativity and vacuum stability conditions when relations in Eq. (17) are applied.

We begin our scan over the parameter space by generating random values of order $\sim 10^{5}$ for three free parameters in the ranges: $1 \mathrm{GeV}<m_{\chi}<1 \mathrm{TeV}, 20 \mathrm{GeV}$ $<m_{h^{\prime}}<150 \mathrm{GeV}$ and $0.01<g^{\prime}<1$. Given the mass relation $m_{Z^{\prime}}=g^{\prime} v^{\prime} / \sqrt{2}$, the $Z^{\prime}$ boson mass will then lie in the range $5.6 \mathrm{GeV}<Z^{\prime}<565 \mathrm{GeV}$. We then use the combined results from Planck [1] and WMAP [2], $0.1172<\Omega h^{2}<0.1226$, to exclude large regions in the parameter space which are inconsistent with these observations. At the same time, when $m_{Z^{\prime}}, m_{h^{\prime}}<m_{h} / 2$ we check further to make sure each generated point in the parameter space can fulfill the upper limit constraint on the invisible Higgs decay width. 

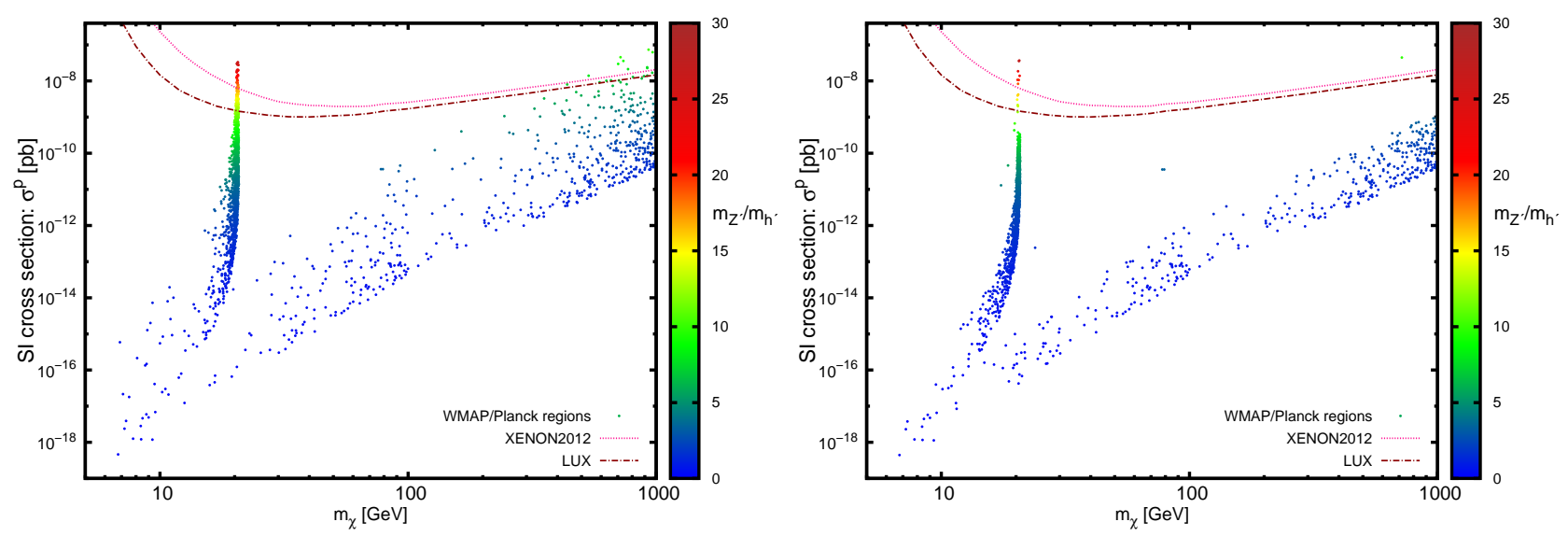

Figure 5: Spin-independent elastic scattering cross section of DM with proton is shown as a function of DM mass. All the points displayed in the plots respect constraints from observed relic density and invisible Higgs decay width measurement. The anticipated upper limit bounds on the elastic scattering cross section imposed by LUX and XENON100 experiments are placed to make comparison. The mixing angle is chosen $\sin \theta=0.01$ in the left panel and $\sin \theta=0.1$ in the right panel.

Using the formula provided by Eq. (28) we compute the DM-proton elastic scattering cross section in terms of DM mass within the parameter space restricted by the observed relic density and invisible Higgs decay width measurement. For a wide range of the DM mass our results for the elastic scattering cross section are summarized in

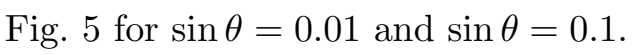

It is evident from these figures that in our model there exist viable regions in the parameter space with DM elastic scattering cross section well below LUX and XENON100 bounds when the ratio $m_{Z^{\prime}} / m_{h^{\prime}} \lesssim 5$. With $\sin \theta=0.01$, all the points with correct relic abundance in the left panel of Fig. 5 respect the upper bound from the invisible Higgs decay. However, for larger mixing angle $\sin \theta=0.1$, the invisible Higgs decay constraint excludes some portion of the region with correct relic density as can be seen by the right panel in Fig. 5 .

Therefore we emphasize here on an interesting feature of the two-portal model that the dark particle can evade direct detection in the range of DM mass from a few $\mathrm{GeV}$ up to $1 \mathrm{TeV}$.

\section{Gamma-Ray Emission From DM Annihila- tion}

The gamma-ray excess observed in the GC from the analyses of the Fermi Large Area Telescope (Fermi-LAT) data is one of the places to look for the trace of the dark matter signals. Among other disfavored scenarios such as millisecond pulsars and cosmic-rays sources, the annihilation of the dark matter (which is more accumulated in the center of the Galaxy) into SM particles explains well the observed gamma-ray 


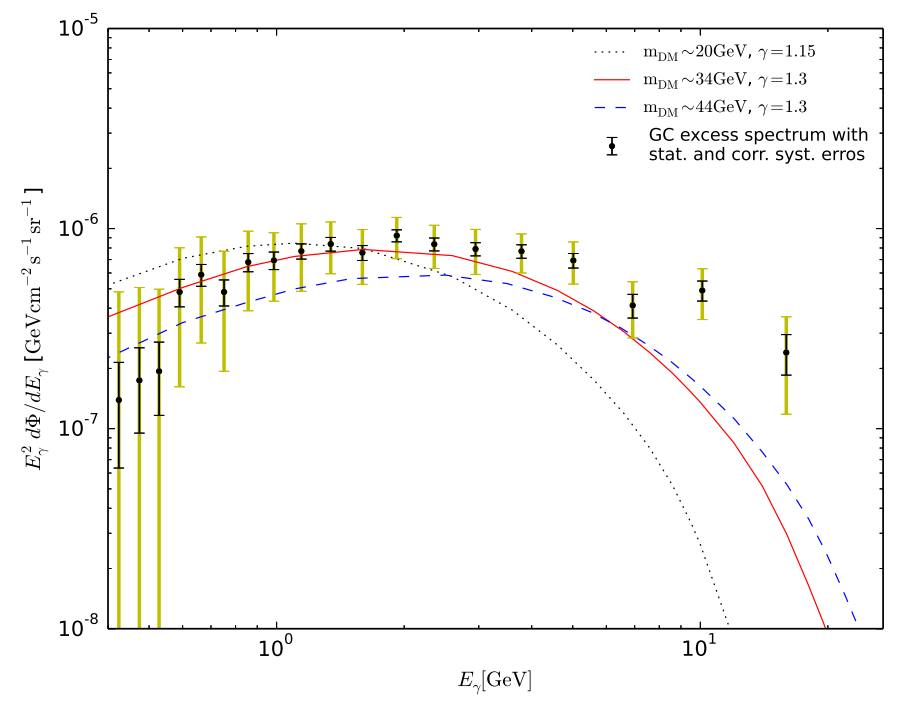

Figure 6: The spectrum of the gamma-ray produced by DM annihilation for masses $m_{\mathrm{DM}}=$ 20,34 and $44 \mathrm{GeV}$ with slop parameter $\gamma=1.15,1,3$ and 1.3 respectively for each DM mass. The DM mass $m_{\mathrm{DM}}=34 \mathrm{GeV}$ is more compatible with Fermi-LAT data analysis [10].

excess.

After it was worked out in [5] where the excess reported for the first time, more accurate analyses were implemented by different groups confirming the original results $[6,10,55]$.

In this section we examine the two-portal model discussed in the last sections for the gamma-ray excess. The region of interest (ROI) we use in our computation is that of considered in [10, i.e. at Galactic latitudes $2^{\circ} \leq|b| \leq 20^{\circ}$ and Galactic longitudes $|l| \leq 20^{\circ}$ known as Inner Galaxy.

Let us briefly review the material we use to obtain the gamma-ray spectrum from dark matter annihilation.

The flux of the gamma-ray produced by annihilation of dark matter into SM particles is given by

$$
\Phi\left(E_{\gamma}, \psi\right)=\frac{\langle\sigma v\rangle}{8 \pi m_{\mathrm{DM}}^{2}} \frac{d N_{\gamma}}{d E_{\gamma}} \int_{\text {l.o.s }} \rho^{2}(r) d l,
$$

where $\langle\sigma v\rangle$ is the velocity averaged total annihilation cross section, $m_{\mathrm{DM}}$ denotes the mass of the dark matter and $d N_{\gamma} / d E_{\gamma}$ is the gamma energy spectrum produced per annihilation. The integral of the density squared is performed over the line-of-sight (l.o.s). The dark matter density as a function of $r$, the distance from the center of the Galaxy, is given by $\rho(r)$. This density function is assumed to be spherically symmetric and is given by the generalized Navarro-Frenk-While (NFW) halo profile [56,57,

$$
\rho(r)=\rho_{0} \frac{\left(r / r_{s}\right)^{-\gamma}}{\left(1+r / r_{s}\right)^{3-\gamma}},
$$


with the local dark matter density $\rho_{0}=0.4 \mathrm{GeV} / \mathrm{cm}^{3}$ and the radius scale $r_{s}=20$ kpc. Due to high uncertainty in the dark matter density near the center of our Milky Way galaxy, the inner slop parameter takes values in the range $\gamma=1-1.3$.

We use micrOMEGAs to compute the gamma-ray spectrum for dark masses $m_{\mathrm{DM}} \sim 20,34,44 \mathrm{GeV}$ that are picked out from the viable parameter space obtained in the previous section (see Fig. [5). The gamma slop parameter is chosen $\gamma=1.15$ for $m_{\mathrm{DM}}=20 \mathrm{GeV}$ and $\gamma=1.3$ for $m_{\mathrm{DM}}=34,44 \mathrm{GeV}$. The annihilation cross section that we obtain for different masses are $\langle\sigma v\rangle=2.14 \times 10^{-26} \mathrm{~cm}^{3} \mathrm{~s}^{-1}$ for $m_{\mathrm{DM}}=20$ $\mathrm{GeV},\langle\sigma v\rangle=2.41 \times 10^{-26} \mathrm{~cm}^{3} \mathrm{~s}^{-1}$ for $m_{\mathrm{DM}}=34 \mathrm{GeV}$ and $\langle\sigma v\rangle=2.34 \times 10^{-26}$ $\mathrm{cm}^{3} \mathrm{~s}^{-1}$ for $m_{\mathrm{DM}}=44 \mathrm{GeV}$. In Fig. 6] we have plotted the energy spectrum of the gamma production for the masses mentioned above. As seen in this figure the DM mass $m_{\mathrm{DM}}=34 \mathrm{GeV}$ has a better agreement with the Fermi-LAT data analysis performed in [10].

\section{Conclusions}

In this paper we have proposed a minimal fermionic dark matter model with two Higgs and vector portals in tandem, both charged under a $U(1)^{\prime}$ symmetry. The $Z^{\prime}$ dark boson beside coupling to the fermionic DM has leptophobic interaction with the SM particles. The $Z^{\prime}$ coupling to the light quarks has also been considered negligible, resulting in a suppressed DM-nucleon interaction. The leading contribution to the DM-nucleon elastic scattering comes from a one-loop Feynman diagram as shown in Fig. 2.

An interesting result is that we find a wide range of DM mass from a few $\mathrm{GeV}$ up to $1 \mathrm{TeV}$ in the viable parameter space respecting all constraints from observed relic abundance, direct detection bounds and invisible Higgs decay width.

The dark matter annihilation into SM particles results in the gamma-ray energy spectrum that fits best with the Fermi-LAT data for the DM mass $m_{\mathrm{DM}}=34 \mathrm{GeV}$.

The new aspects of the two-portal DM in comparison with the earlier works which involve either the Higgs portal or the $Z^{\prime}$ portal are discussed below.

1) In the fermionic DM model introduced in [58, DM interacts with the SM particles with exchanging the SM Higgs $(h)$ or a new scalar particle $\left(h^{\prime}\right)$. They found out that almost the entire parameter space is excluded by the XENON and CDMS except a very small resonant regions corresponding to very restricted conditions for the DM mass: $m_{\chi} \sim m_{h} / 2$ or $m_{\chi} \sim m_{h^{\prime}} / 2$. It is likely that with future experiments like XENON1T, these small viable regions get even smaller. Moreover, the model discussed in 58] predicts a large invisible Higgs decay width, such that with the current bound from the LHC, the DM masses less than about $50 \mathrm{GeV}$ are excluded. In contrast, the presented model in this paper with simultaneous two portals provides us with a rather wide viable parameter space well below XENON100 and LUX bounds. More precisely, we see that the viable range for the DM mass is from a few GeV up to $1 \mathrm{TeV}$. It is quite unlikely that the future proposed or planned direct detection experiments can rule out the entire viable parameter space for the aforementioned 
range of the DM mass. One more interesting feature of the proposed model is that the total Higgs decay width including the effects of the new particles does not depend on the DM mass. Therefore, even for small DM mass it becomes possible to find viable regions in the parameter space which respect the invisible Higgs decay width bound.

2) In the two-portal model, the coupling between the fermionic DM and the $Z^{\prime}$ boson is of vector type while the anomaly free condition imposes a pure axial coupling between the $Z^{\prime}$ boson with the third family of quarks. One crucial feature of the present model is the fact that with the help of scalar mediator (Higgs portal) and the non-universality of the $Z^{\prime}$ boson, spin-independent elastic scattering of the DM with nuclei is possible. In the earlier works (see e.g. [26, 27]) with the same axial coupling between $Z^{\prime}$ boson and the third family of quarks there is no DM-nucleon elastic scattering at all. Therefore we have shown in the two-portal model that even without having any $Z^{\prime}$ interaction with $u$ and $d$ quarks, there is a prospect for a signal at the direct detection experiments.

\section{A Dark Matter Annihilation Cross Sections}

We give the DM annihilation cross section formulas in this section. The annihilation cross section for the process $\chi \chi \rightarrow \bar{f} f$ with $f=b, t$ is obtained as

$$
\sigma_{\mathrm{ann}} v_{\mathrm{rel}}(\bar{\chi} \chi \rightarrow \bar{f} f)=\frac{g^{\prime 4} \sqrt{1-4 m_{f}^{2} / s}}{2 \pi s}\left[\frac{s^{2}-8 m_{f}^{2} m_{\chi}^{2}+2 s m_{\chi}^{2}-\frac{4}{9} s m_{f}^{2}}{\left(s-m_{Z^{\prime}}^{2}\right)^{2}+m_{Z^{\prime}}^{2} \Gamma_{Z^{\prime}}^{2}}\right] .
$$

The next annihilation process $\chi \chi \rightarrow h Z^{\prime}$, is again mediated by a dark gauge boson via s-channel. We get the following result for the cross section as,

$\sigma_{\text {ann }} v_{\text {rel }}\left(\bar{\chi} \chi \rightarrow h Z^{\prime}\right)=\frac{g^{\prime} v^{\prime 2} \sin ^{2} \theta}{16 \pi s} \frac{\left(s+2 m_{\chi}^{2}\right) \sqrt{\left[1-\left(m_{h}^{2}+m_{Z^{\prime}}^{2}\right) / s\right]^{2}-4 m_{h}^{2} m_{Z^{\prime}}^{2} / s^{2}}}{\left(s-m_{Z^{\prime}}^{2}\right)^{2}+m_{Z^{\prime}}^{2} \Gamma_{Z^{\prime}}^{2}}$.

We then obtain the DM annihilation cross section for the process $\chi \chi \rightarrow h^{\prime} Z^{\prime}$,

$\sigma_{\mathrm{ann}} v_{\mathrm{rel}}\left(\bar{\chi} \chi \rightarrow h^{\prime} Z^{\prime}\right)=\frac{g^{\prime} v^{\prime 2} \cos ^{2} \theta}{16 \pi s} \frac{\left(s+2 m_{\chi}^{2}\right) \sqrt{\left[1-\left(m_{h^{\prime}}^{2}+m_{Z^{\prime}}^{2}\right) / s\right]^{2}-4 m_{h^{\prime}}^{2} m_{Z^{\prime}}^{2} / s^{2}}}{\left(s-m_{Z^{\prime}}^{2}\right)^{2}+m_{Z^{\prime}}^{2} \Gamma_{Z^{\prime}}^{2}}$

Finally, we get the annihilation cross section for the process $\chi \chi \rightarrow Z^{\prime} Z^{\prime}$ which takes place by mediating a DM via t- and u-channel,

$$
\begin{array}{r}
\sigma_{\mathrm{ann}} v_{\mathrm{rel}}\left(\bar{\chi} \chi \rightarrow Z^{\prime} Z^{\prime}\right)=\frac{g^{\prime 4} \sqrt{1-4 m_{Z^{\prime}}^{2} / s}}{8 \pi^{2} s} \int d \Omega\left[\frac{s m_{Z^{\prime}}^{2}-m_{\chi}^{2} m_{Z^{\prime}}^{2}+\frac{1}{2} s m_{\chi}^{2}-2 m_{\chi}^{4}}{\left(t-m_{\chi}^{2}\right)\left(u-m_{\chi}^{2}\right)}\right. \\
-\frac{\left(m_{\chi}^{2}+m_{Z^{\prime}}^{2}-t\right)^{2}+t s-s m_{\chi}^{2}+2 t m_{\chi}^{2}+4 m_{\chi}^{2} m_{Z^{\prime}}^{2}+2 m_{\chi}^{4}}{2\left(t-m_{\chi}^{2}\right)^{2}} \\
\left.-\frac{\left(m_{\chi}^{2}+m_{Z^{\prime}}^{2}-u\right)^{2}+u s-s m_{\chi}^{2}+2 u m_{\chi}^{2}+4 m_{\chi}^{2} m_{Z^{\prime}}^{2}+2 m_{\chi}^{4}}{2\left(u-m_{\chi}^{2}\right)^{2}}\right]
\end{array}
$$

where, $s, t$ and $u$ are the relevant mandelstam variables. 


\section{References}

[1] P. A. R. Ade et al. [Planck Collaboration], Astron. Astrophys. 571 (2014) A16 arXiv:1303.5076 [astro-ph.CO]].

[2] G. Hinshaw et al. [WMAP Collaboration], Astrophys. J. Suppl. 208 (2013) 19 arXiv:1212.5226 [astro-ph.CO]].

[3] D. S. Akerib et al. [LUX Collaboration], Phys. Rev. Lett. 112 (2014) 091303 arXiv:1310.8214 [astro-ph.CO]].

[4] E. Aprile et al. [XENON100 Collaboration], Phys. Rev. Lett. 109 (2012) 181301 arXiv:1207.5988 [astro-ph.CO]].

[5] D. Hooper and L. Goodenough, Phys. Lett. B 697 (2011) 412 arXiv:1010.2752 [hep-ph]].

[6] K. N. Abazajian and M. Kaplinghat, Phys. Rev. D 86 (2012) 083511

[7] C. Gordon and O. Macias, Phys. Rev. D 88 (2013) 083521 arXiv:1306.5725 [astro-ph.HE]].

[8] K. N. Abazajian, N. Canac, S. Horiuchi and M. Kaplinghat, Phys. Rev. D 90 (2014) 023526 [arXiv:1402.4090 [astro-ph.HE]].

[9] T. Daylan, D. P. Finkbeiner, D. Hooper, T. Linden, S. K. N. Portillo, N. L. Rodd and T. R. Slatyer, arXiv:1402.6703 [astro-ph.HE].

[10] F. Calore, I. Cholis and C. Weniger, arXiv:1409.0042 [astro-ph.CO].

[11] F. Calore, I. Cholis, C. McCabe and C. Weniger, Phys. Rev. D 91 (2015) 6, 063003 [arXiv:1411.4647 [hep-ph]].

[12] C. Boehm, M. J. Dolan, C. McCabe, M. Spannowsky and C. J. Wallace, JCAP 1405 (2014) 009 arXiv:1401.6458 [hep-ph]].

[13] S. Ipek, D. McKeen and A. E. Nelson, Phys. Rev. D 90 (2014) 5, 055021 arXiv:1404.3716 [hep-ph]].

[14] E. Izaguirre, G. Krnjaic and B. Shuve, Phys. Rev. D 90 (2014) 5, 055002 arXiv:1404.2018 [hep-ph]].

[15] C. Cheung, M. Papucci, D. Sanford, N. R. Shah and K. M. Zurek, Phys. Rev. D 90 (2014) 7, 075011 [arXiv:1406.6372 [hep-ph]].

[16] K. Ghorbani, JCAP 1501 (2015) 015 [arXiv:1408.4929 [hep-ph]].

[17] M. J. Dolan, C. McCabe, F. Kahlhoefer and K. Schmidt-Hoberg, JHEP 1503 (2015) 171 arXiv:1412.5174 [hep-ph]].

[18] T. Gherghetta, B. von Harling, A. D. Medina, M. A. Schmidt and T. Trott, arXiv:1502.07173 [hep-ph].

[19] E. Dudas, Y. Mambrini, S. Pokorski and A. Romagnoni, JHEP 0908 (2009) 014 arXiv:0904.1745 [hep-ph]].

[20] X. Chu, Y. Mambrini, J. Quevillon and B. Zaldivar, JCAP 1401 (2014) 01, 034 arXiv:1306.4677 [hep-ph]]. 
[21] G. Arcadi, Y. Mambrini, M. H. G. Tytgat and B. Zaldivar, JHEP 1403 (2014) 134 [arXiv:1401.0221 [hep-ph]].

[22] O. Lebedev and Y. Mambrini, Phys. Lett. B 734 (2014) 350 arXiv:1403.4837 [hep-ph]].

[23] G. Belanger, A. Pukhov and G. Servant, JCAP 0801 (2008) 009 arXiv:0706.0526 [hep-ph]].

[24] M. R. Buckley, D. Hooper and J. L. Rosner, Phys. Lett. B 703 (2011) 343 arXiv:1106.3583 [hep-ph]].

[25] M. Pospelov, A. Ritz and M. B. Voloshin, Phys. Lett. B 662 (2008) 53 arXiv:0711.4866 [hep-ph]].

[26] D. Hooper, Phys. Rev. D 91 (2015) 3, 035025 [arXiv:1411.4079 [hep-ph]].

[27] A. Berlin, D. Hooper and S. D. McDermott, Phys. Rev. D 89 (2014) 11, 115022 arXiv:1404.0022 [hep-ph]].

[28] J. K. Mizukoshi, C. A. de S.Pires, F. S. Queiroz and P. S. Rodrigues da Silva, Phys. Rev. D 83 (2011) 065024 [arXiv:1010.4097 [hep-ph]].

[29] A. Alves, A. Berlin, S. Profumo and F. S. Queiroz, arXiv:1501.03490 [hep-ph].

[30] D. London and J. L. Rosner, Phys. Rev. D 34, 1530 (1986).

[31] J. L. Hewett and T. G. Rizzo, Phys. Rept. 183 (1989) 193.

[32] P. Langacker, Rev. Mod. Phys. 81 (2009) 1199 [arXiv:0801.1345 [hep-ph]].

[33] C. T. Hill and E. H. Simmons, Phys. Rept. 381 (2003) 235 [Phys. Rept. 390 (2004) 553] hep-ph/0203079.

[34] F. del Aguila, G. A. Blair, M. Daniel and G. G. Ross, Nucl. Phys. B 283 (1987) 50.

[35] N. Arkani-Hamed, A. G. Cohen and H. Georgi, Phys. Lett. B 516 (2001) 395 hep-th/0103135.

[36] N. Arkani-Hamed, A. G. Cohen, E. Katz, A. E. Nelson, T. Gregoire and J. G. Wacker, JHEP 0208 (2002) 021 hep-ph/0206020.

[37] T. Han, H. E. Logan, B. McElrath and L. T. Wang, Phys. Rev. D 67 (2003) 095004 hep-ph/0301040].

[38] M. Perelstein, Prog. Part. Nucl. Phys. 58 (2007) 247 hep-ph/0512128].

[39] Z. Chacko, H. S. Goh and R. Harnik, Phys. Rev. Lett. 96 (2006) 231802 hep-ph/0506256.

[40] D. A. Demir, G. L. Kane and T. T. Wang, Phys. Rev. D 72 (2005) 015012 hep-ph/0503290.

[41] K. Ghorbani and H. Ghorbani, arXiv:1501.00206 [hep-ph].

[42] J. M. Cline, G. Dupuis, Z. Liu and W. Xue, arXiv:1503.08213 [hep-ph].

[43] C. T. Hill, Phys. Lett. B 345 (1995) 483 hep-ph/9411426. 
[44] A. A. Andrianov, P. Osland, A. A. Pankov, N. V. Romanenko and J. Sirkka, Phys. Rev. D 58 (1998) 075001 hep-ph/9804389].

[45] R. S. Chivukula and E. H. Simmons, Phys. Rev. D 66 (2002) 015006 hep-ph/0205064.

[46] J. Y. Liu, Y. Tang and Y. L. Wu, J. Phys. G 39 (2012) 055003 arXiv:1108.5012 [hep-ph]].

[47] S. Heinemeyer et al. [LHC Higgs Cross Section Working Group Collaboration], arXiv:1307.1347 [hep-ph].

[48] G. Belanger, B. Dumont, U. Ellwanger, J. F. Gunion and S. Kraml, Phys. Lett. B 723 (2013) 340 arXiv:1302.5694 [hep-ph]].

[49] G. Belanger, F. Boudjema, A. Pukhov and A. Semenov, Comput. Phys. Commun. 180 (2009) 747 [arXiv:0803.2360 [hep-ph]].

[50] J. R. Ellis, K. A. Olive and C. Savage, Phys. Rev. D 77 (2008) 065026 arXiv:0801.3656 [hep-ph]].

[51] T. Nihei and M. Sasagawa, Phys. Rev. D 70 (2004) 055011 [Erratum-ibid. D 70 (2004) 079901] [hep-ph/0404100].

[52] G. Belanger, F. Boudjema, A. Pukhov and A. Semenov, Comput. Phys. Commun. 185 (2014) 960 [arXiv:1305.0237 [hep-ph]].

[53] A. Semenov, arXiv:1005.1909 [hep-ph].

[54] A. Belyaev, N. D. Christensen and A. Pukhov, Comput. Phys. Commun. 184 (2013) 1729 arXiv:1207.6082 [hep-ph]].

[55] O. Macias and C. Gordon, Phys. Rev. D 89 (2014) 6, 063515 arXiv:1312.6671 [astro-ph.HE]].

[56] J. F. Navarro, C. S. Frenk and S. D. M. White, Astrophys. J. 462 (1996) 563 astro-ph/9508025.

[57] J. F. Navarro, C. S. Frenk and S. D. M. White, Astrophys. J. 490 (1997) 493 astro-ph/9611107.

[58] Y. G. Kim, K. Y. Lee and S. Shin, JHEP 0805 (2008) 100 arXiv:0803.2932 [hep-ph]]. 